\title{
M2 receptors are required for spatiotemporal sequence learning in mouse primary visual cortex
}

\author{
Authors \\ Susrita Sarkar ${ }^{1}$, Catalina Martinez Reyes ${ }^{1}$, Cambria M. Jensen ${ }^{1}$, and Jeffrey P. Gavornik ${ }^{1,2}$ \\ Institutional Affiliations \\ ${ }^{1}$ Center for Systems Neuroscience, Department of Biology, Boston University, Boston, MA 02215. \\ ${ }^{2}$ Correspondence should be sent to gavornik@bu.edu
}

\begin{abstract}
Acetylcholine (ACh) is a neurotransmitter that plays a variety of roles in the central nervous system (CNS). It was previously shown that blocking muscarinic receptors with a non-selective antagonist prevents a form of experiencedependent plasticity termed "spatiotemporal sequence learning" in the mouse primary visual cortex (V1). Muscarinic signaling is a complex process involving the combined activities of five different G-protein coupled receptors, M1M5, all of which are expressed in the murine brain but differ from each other functionally and in anatomical localization. Here we present electrophysiological evidence that M2, but not M1, receptors are required for spatiotemporal sequence learning in mouse V1. We show that M2 is highly expressed in the neuropil in V1, especially in thalamorecipient layer 4, and co-localizes to the soma in a subset of somatostatin expressing neurons in deep layers. We also show that expression of M2 receptors is higher in the monocular region of V1 than it is in the binocular region, but that the amount of experience-dependent sequence potentiation is similar in both regions. This work establishes a new functional role for M2-type receptors in processing temporal information and demonstrates that monocular circuits are modified by experience in a manner similar to binocular circuits.
\end{abstract}

\section{Acknowledgements}

We thank all the mice that gave their lives for this research, without their sacrifice this paper wouldn't have existed. We thank Gianna Ferron of the Gavornik Lab for her support running experiments, and Todd Blute of the Boston University Biology Imaging Core for help using the fluorescence microscope. Finally, thanks to the great folks at Microsoft who after decades of tireless work still don't know how to make a word processing program where it is possible to cut/paste without completely destroying all of the formatting. This work was supported by grant NEI R01EY030200. 


\section{Introduction}

Acetylcholine $(\mathrm{ACh})$ is a neurotransmitter and neuromodulator in the brain involved with a wide range of functions including attention (Herrero et al., 2008; Parikh et al., 2007; Sarter et al., 2005), motivation (Collins et al., 2016), aggression (Lewis et al., 2018), thermoregulation (Beckman and Carlisle, 1969), appetitive behavior (Herman et al., 2016), sexual behavior (Floody, 2014), sleep-wake cycle (Hasselmo and McGaughy, 2004; Steriade, 2004; Lee and Dan, 2012), memory formation (Hasselmo, 2006), sensory perception (Pinto et al., 2013; Goard \& Dan, 2009), temporal processing (Gavornik and Bear, 2014), plasticity (Bear and Singer, 1986), and synchronizing rhythmic oscillations (Gu, 2003; Hasselmo and McGaughy, 2004; Steriade, 2004; Thiele, 2013). The complex relationship between cholinergic signaling and neural activity is attributed to differences in the function and anatomical distribution of nicotinic (nAChR) and muscarinic (mAChR) receptors. Of interest here are the mAChRs which are required for a form of experience-dependent plasticity that can encode spatiotemporal information in mouse V1 (Gavornik and Bear, 2014) and other areas as well (Finnie, et al., 2021; Sidorov et al., 2020).

mAChRs are heptahelical metabotropic G-protein coupled receptors (GPCRs) and are classified into five subtypes (M1-M5) which are functionally condensed into two group types based on the family of G-proteins they are coupled to. M1-type receptors (M1, M3 and M5) are associated with the $\mathrm{G}_{\mathrm{q} / 11}$ family whereas M2-type receptors (M2 and M4) are coupled to the $\mathrm{G}_{\mathrm{i} / \mathrm{o}}$ family (Thiele, 2013; Groleau et al., 2015; Coppola and Disney, 2018). M1, M2 and M4 are the most abundant receptors in the cortex, striatum, thalamus, and hippocampus with the cortex expressing more M2 than M1 (Bubser et al., 2012.; Levey et al., 1991; Thiele, 2013; Volpicelli \& Levey, 2004). M1-type receptors are predominantly post-synaptic whereas M2-type receptors are generally presynaptic, though all five isoforms can be found both at pre- and post-synaptic sites (Thiele, 2013; Groleau et al., 2015). M2-type receptors can be found on cholinergic terminals where they act as auto receptors preventing further release of ACh when the extracellular concentration of ACh is high (Mrzljak et al., 1993; Groleau et al., 2015), and they can also prevent the release of GABA from GABAergic terminals (Salgado et al., 2007) under similar conditions. M2 receptors are found in almost all layers of mouse V1 but are primarily concentrated in L4 (Vaucher et al., 2002; Ji et al., 2015). Individual studies have reported information about the cell-type and layer-specific localization of muscarinic receptors in V1, though not all those studies were done in mice.

Scopolamine is a non-selective antagonist that blocks M1-M5 (Lochner and Thompson, 2016). In a 2014 study, Gavornik and Bear showed that binocular V1 can encode a prediction of both the sequential order and element timing 
contained in visual sequences, but that the potentiation associated with this plasticity is blocked following systemic or local-to-V1 treatment with scopolamine. This finding directly implicates cholinergic signaling in a form of experience-dependent plasticity that is specific in both the spatial and temporal domains but is not the only one showing that ACh can entrain temporal representations in V1. Cholinergic inputs from the basal forebrain (BF), which is the principal source of ACh for the neocortex (Thiele, 2013), have also been shown to allow V1 circuits to report temporal intervals between a flashed visual stimulus and reward signal in rodent V1 using both in vivo (Shuler \& Bear, 2006; Liu et al., 2015; Namboodiri et al., 2015) and ex vivo preparations (Chubykin et al., 2013). Despite these works, it is an open question how cholinergic signaling helps encode temporal or ordinal ordering in cortical circuits. Given the relationship between location within the cortical column and function (Muñoz \& Rudy, 2014), as well as the diverse function repertoire of mAChRs and neural cell-types, it is important to determine which receptors are required for spatiotemporal sequence learning and where they are located to mechanistically characterize the circuitry and biochemical signaling involved in sequence learning.

Here we employ the same "sequence learning" paradigm used in Gavornik and Bear, 2014. Briefly, this form of plasticity causes the responses evoked in V1 by a 4-element visual sequence to potentiate with exposure and is somewhat selective for the temporal order of sequence elements (e.g., evoked potentials get smaller when the elements are reordered). We find that muscarinic receptor M2, but not M1 is required for spatiotemporal sequence learning in mouse V1. We also report on the layer-specific distribution of M2 receptors in V1. Most of the expression is found in the neuropil, but we show that these receptors are also expressed somatically on some somatostatin (SST) positive neurons in deep cortical layers. We also find that M2 expression is higher in monocular V1 than binocular V1. Finally, we show that sequence learning occurs in monocular V1 with potentiation magnitude similar to that in binocular V1 despite the increased M2 levels in monocular cortex.

\section{Results}

\section{M1 receptors are not required for spatiotemporal sequence learning in mouse V1.}

Though there are five muscarinic receptors not all are expressed in rodent V1. Of the M1-type receptors only M1 is abundant, M3 is not detectable in rodent V1 via immunocytochemistry (Groleau et al., 2015; Levey et al., 1994) and M5 is found primarily on endothelial cells with minimal expression in rodent visual cortex (Elhusseiny and Hamel, 2000; Groleau et al., 2015). Both M2-type receptors are found in mouse brain with M2 dominant in the cortex and M4 
in the striatum (Groleau et al., 2015; Bubser et al., 2012; Levey et al., 1991; Thiele, 2013; Zhang et al., 2002; Flynn et al., 1995). M1, which is generally excitatory, seemed more likely to be responsible for potentiated responses associated with spatiotemporal plasticity. Accordingly, for our first experiment we used chronically implanted electrodes to record visually evoked potentials (VEPs) from binocular layer 4 of mouse V1 in response to a sequence stimulus while pharmacologically blocking the M1 receptor. Following Gavornik and Bear, mice were trained for 4 days via passive exposure (Fig. 1A) to 200 presentations of a four-element sequence, $\mathrm{ABCD}$, where each element was a sinusoidal grating with a unique orientation. Each element was held on the screen for 150 $\mathrm{ms}$, for a total sequence time of $600 \mathrm{~ms}$, and sequences were separated by $1.5 \mathrm{~s}$ of gray
A.
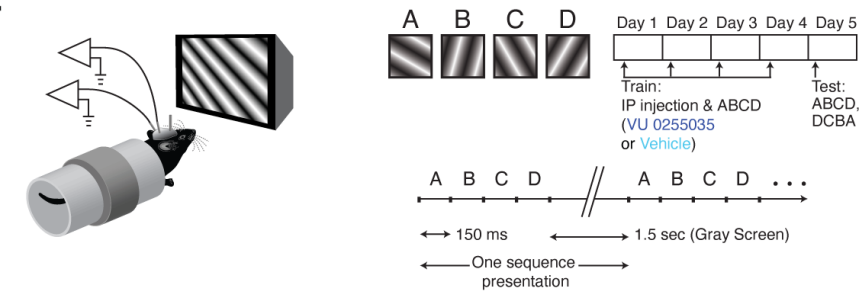

B.

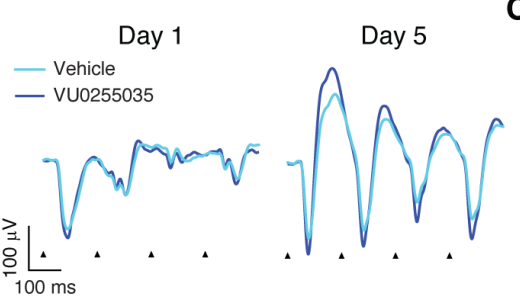

C.

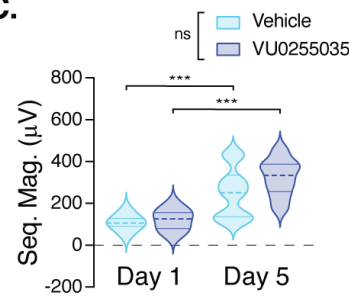

Figure $1 \mathrm{M} 1$ receptors are not required for spatiotemporal sequence learning in V1. A. Mice were injected with either the M1 receptor antagonist VU 0255035 (exp, $n=8$ ) or vehicle (ctrl, $n=5$ ) $\sim 30$ mins prior to head-fixed stimulus presentation on each of four training days. Training consisted of 200 presentations of the sequence ABCD. All mice were tested without injection on the fifth day with no injections using same sequence ABCD (and DCBA, see Fig. S1). B. Sequence-evoked local field potentials looked very similar in drug and vehicle-treated animals at baseline (day 1) and the responses potentiated a similar amount in both groups after training (day 5). Voltage traces here and in subsequent figures represent stimulus locked LFP response averaged across all mice in each group, triangles mark the onset of each stimulus in the sequence. C. Sequence magnitudes (quantified as the average peak-to-peak response to each element) recorded on days 1 and 5 were analyzed using a mixed model RM-ANOVA which showed a significant effect of recording day $\left(F_{1,11}=71.659, p<0.001\right)$ but no effect of treatment $\left(F_{1,11}=10.71, p=0.323\right)$ or significant interaction between treatment and day $\left(F_{1,11}=2.272, p=0.160\right)$. Post-hoc analysis with a Bonferroni correction revealed that sequence magnitude was significantly larger on day 5 than day 1 in both drug $(202.1 \mu \mathrm{V}, \mathrm{p}<$ $0.001)$ and vehicle $(141.0 \mu \mathrm{V}, p=0.001)$ treated animals. Quantified data is visualized using violin plots whose shapes represent an estimate of the probability density distribution (solid horizontal lines mark the first and third quartile divisions, the dashed line shows the median).

screen. Mice were randomly assigned to yoked experimental and control groups. On each training day, mice in the experimental group $(\mathrm{n}=8)$ were injected intraperitoneally with a highly selective M1 antagonist capable of crossing the blood brain barrier, VU 0255035 (Weaver et al., 2009), 30 mins before stimulus presentation. Mice in the control group $(n=5)$ were similarly injected with vehicle. On the test day (day 5) mice in both groups were presented with the same stimulus without a prior injection of VU 0255035 or vehicle. VEP magnitudes increased with training in both groups and were markedly larger on day 5 than on day 1 (Fig. 1B) with no obvious difference between groups on any recording day. To quantify sequence responses, we calculated the peak-to-peak VEP magnitude after each element's presentation and averaged these values together to create a sequence magnitude value (see methods). In accord with 
our observation about the shape of evoked responses, we find no significant difference between quantified sequence magnitudes (Fig. 1C, Fig. S1) as a function of treatment group, demonstrating that M1 receptors are not required for spatiotemporal sequence potentiation in mouse V1.

\section{M2 receptors are required for spatiotemporal sequence learning in mouse} V1.

Our next experiment was to block M2 receptors using a high affinity, selective M2 antagonist AQ-RA 741 (Doods et al., 1991; Dorje et al., 1991). Under physiological conditions, AQ-RA 741 is water soluble because of double protonation and, unlike VU 0255035, is unable to cross the blood brain barrier (Doods et al., 1993). Accordingly, we modified our experimental design to infuse the drug directly into V1 via indwelling canulae (Fig. 2A) while recording sequence VEPs from binocular layer 4 of mouse V1. As before, mice were randomly assigned to experimental and yoked control groups. On each training day, mice in the experimental group $(n=12)$ were intracerebrally infused with AQ-RA 741 and mice in the control group $(\mathrm{n}=11)$ received vehicle infusion under light isoflurane anesthesia and exposed to stimulation $\sim 30$ mins after infusion completion. To minimize complications associated with multiple
A.

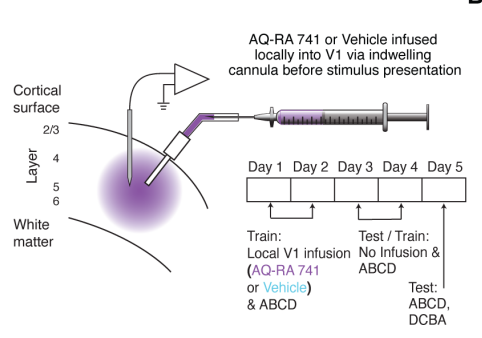

B.

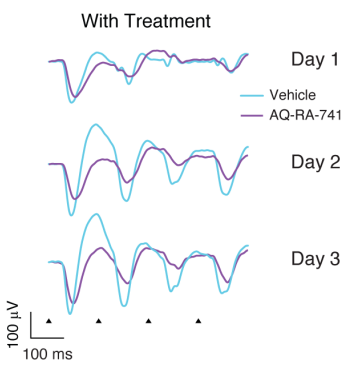

c.

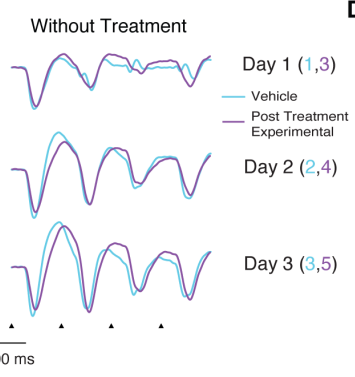

D.

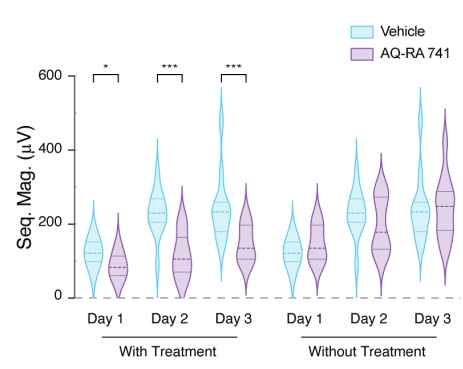

Figure 2 M2 receptors are required for spatiotemporal sequence learning in V1. A. M2 receptors were antagonized through intracerebral infusions of the M2 antagonist AQ-RA 741 via an indwelling cannula surgically implanted in V1. Animals were treated with either drug (exp, $n=12$ ) or vehicle (ctrl, $n=11$ ) under isoflurane anesthesia $\sim 30$ mins before stimulus presentation on training days 1 and day 2 but not before test stimuli on day 3. Training continued for an additional day and a final set of test stimuli was presented on day 5 (see also Fig. S2). B. Sequence-evoked responses potentiated normally in vehicle-treated mice but was impaired in drug-treated animals. C. After drug washout, the responses in drug-treated animals potentiated at a level commensurate with vehicle-treated animals. This panel compares responses over the first three days of un-treated training for vehicle and drug treated mice (days 1-3 and 3-5, respectively). D. The effect of training and treatment was analyzed on days 1-3 using a mixed model RM-ANOVA which revealed significant main effects of day $\left(F_{2,42}=\right.$ $33.384, p<0.001)$ and treatment $\left(F_{1,21}=15.788, p=0.001\right)$ and a significant interaction between the two $\left(F_{2,42}=6.200, \mathrm{p}=0.004\right)$. Bonferroni-corrected post-hoc tests showed a small but significant difference between groups on day $1(34.6 \mu \mathrm{V}, \mathrm{p}=0.022)$ that increased on days $2(111.9 \mu \mathrm{V}, \mathrm{p}<0.001)$ and $3(97.6 \mu \mathrm{V}, \mathrm{p}=0.004)$. Responses in drug-treated animals increased on both days 2 and 3 but not significantly (1vs2: $29.6 \mu \mathrm{V}, \mathrm{p}=0.144 .2 \mathrm{vs} 3: 30.8 \mu \mathrm{V}, \mathrm{p}=0.178$ ) whereas vehicle-treated animals showed a large amount of potentiation on day $2(1 \mathrm{vs} 2: 106.9 \mu \mathrm{V}, \mathrm{p}<0.001)$ and modest potentiation on day 3 (2vs3: $16.6 \mu \mathrm{V}, \mathrm{p}=0.946$ ). The effects of training without treatment were similarly analyzed using the comparisons from panel $\mathrm{C}$. This revealed a significant effect of training $\left(F_{2,42}=33.790, p<0.001\right)$ but no effect of treatment group $\left(F_{1,21}=0.009, p=0.924\right)$ or significant interaction $\left(F_{2,42}=2.167, p=0.127\right)$ effect, demonstrating that the lack of potentiation on days 2-3 was an acute effect of drug treatment. 
infusions, we trained animals for only two days before testing without infusion on day 3. Our previous experience and published results show that 2 days is sufficient to see statistically significant different effects of drug treatments in the paradigm. As shown in Fig. 2B, there was minimal change in experimental VEPs over this period while VEPs in control animals potentiated considerably. To make sure that the lack of potentiation in experimental animals was caused by drug action and damage caused by the infusions, we continued to train the experimental cohort for an additional two days. As shown in Fig. 2C, VEPs in experimental animals on day 3 (the first day of non-drug treated visual exposure) were essentially the same as on day 1 in control mice. From day 3 up to day 5 , animals in the experimental group showed normal sequence potentiation to ABCD and the VEPs. While VEP magnitude changes were significantly different between experiment and control animals on days 2 and 3 during drug treatment, there was no significant difference when comparing the groups on the 3rd day of non-treated potentiation (see also Fig. S2). We interpret these results to mean that significant potentiation between day 1 and day 3 in experimental animals was prevented by M2 receptor blockage. Once drug administration was stopped after on day 2, treated tissue regained the capacity to potentiate to $\mathrm{ABCD}$. These findings demonstrate that $\mathrm{M} 2$ receptors are required for spatiotemporal sequence potentiation in mouse $\mathrm{V} 1$.

\section{M2 receptors are differentially expressed based on cortical layer, cell type, and location within V1.}

Since our electrophysiology experiments revealed that M2 receptors are required for sequence learning in mouse V1, we next turned to immunohistochemistry to look in detail at the layer-wise distribution of M2 receptors in V1. We used stained for M2 receptors using immunofluorescence labeling in coronal brain slices acquired from mice agematched to our experimental cohorts. Resulting images show that M2 (Fig. 3A) receptors are found in all layers of mouse V1 with an intensity profile that varies by layer with a marked difference in M2 expression levels between monocular and binocular layer 4. Similar to what was reported by Ji et al., 2015, we see that expression peaks in layer 4 and is also relatively high in layer 5B (Fig. 3B). To make sure that the M2 expression pattern was not attributable to cell density, we also stained for DSDNA (DAPI) in the same coronal slices and verified that the expression patterns did not match (Fig. S4).

While most M2 expression was seen in neuropil, we did observe a small number of cell bodies that were positive for M2 (arrows, Fig 3A,E). Different classes of inhibitory interneurons are functionally implicated in various V1 computations (Chen et al., 2015; Hooks \& Chen, 2020; Pfeffer et al., 2013; Niell and Scanziani, 2021) and we decided 
A.

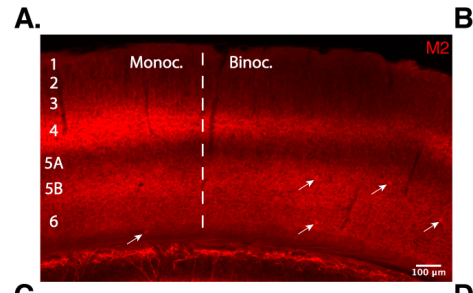

B.
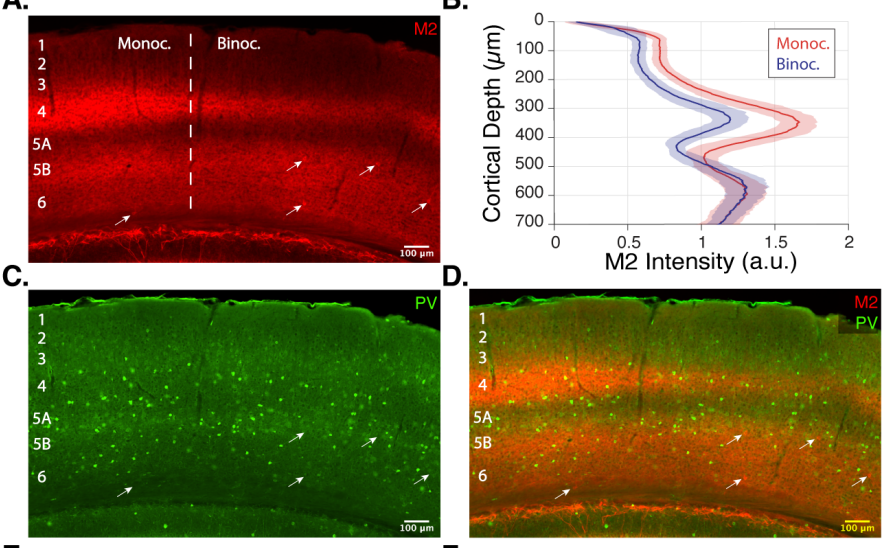

E.
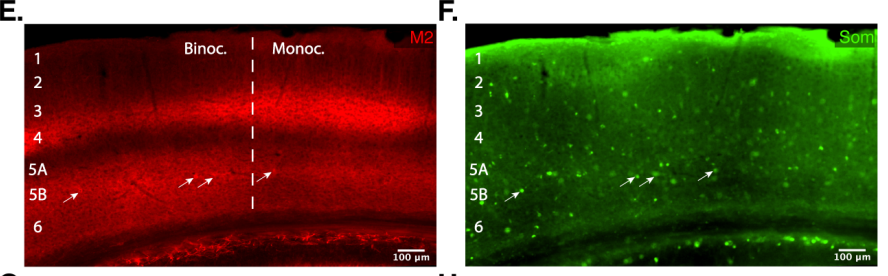

G.

H.

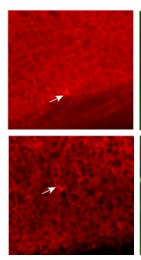

to determine if M2 receptors are present on

Parvalbumin (PV) or SST interneurons by

using immunofluorescence co-labeling for

two different markers, PV or SST and M2.

Resulting images (Fig 3.A,C-D, )

revealed that M2 receptors are not expressed

on cell bodies of PV neurons in mouse V1.

We found approximately 116 PV neurons per

50-micron thick V1 section in mice stained

for PV and M2 (slices from $\mathrm{n}=4$ mice,

$\mu=115.5, \sigma=3.9$ ), none of which were positive

for M2. Double immunolabeling with M2 and

SST antibodies, however, did show that M2

receptors are expressed on cell bodies and

processes of SST neurons in V1(Fig. 3E-G).

Figure 3 M2 receptors are not expressed on cell bodies of PV interneurons in V1 but are expressed on cell bodies of a subset of SST interneurons in V1.A. Representative image of M2 receptor antibody staining showing that M2 receptors are found in all layers of V1. Expression levels vary between layers. The majority of fluorescence originates in the neuropil, though there are a few labeled cell bodies in deep layers (marked with arrows). In addition, there is a clear increase in layer-4 expression in monocular V1 (left) relative to binocular (right, separated by a dashed line, the position of which was determined using hippocampal landmarks identified in the Mouse Brain Atlas). B. M2 depth-wise intensity profiles in monocular and binocular V1 quantify higher expression levels in supragranular (17.9\%) and granular (45.8\%) layers of monocular cortex, while expression in infragranular layers is approximately equal (-1.85\%). Solid lines indicated normalized group mean intensity of M2 expression (slices from $n=9$ mice) with $95 \%$ confidence intervals (shaded areas). A 2-way ANOVA of intensity values shows that there is a significant effect of depth (supra/infra/sub, $F_{2,48}=$ $40.650, p<0.001$ ) and cortical area (monoc/binoc, $F_{1,48}=34.817, p<$ $0.11)$ with a significant interaction between groups $\left(F_{2,48}=12.316, p<\right.$ 0.001). Post-hoc analysis with Bonferroni correction confirms the previous observation that $\mathrm{M} 2$ expression levels are significantly higher monocular V1 than in binocular V1 in layers 1-3 (0.218 a.u., $p=0.001)$ and layer 4 ( 0.428 a.u, $p<0.001)$, but the same in deep layers $(-0.009$ a.u., $p=0.884$ ). C. Cell bodies identified in the M2 channel (arrows) do not match the location of PV+ somas. D. Overlap images of the M2 (red) and PV (green) channels confirm that PV somas do not express M2 receptors. E. A second representative image showing M2 expression (details as in A, though from a different mouse and in the left rather than right hemisphere). F-H. Anti-SST co-labeling of the same slice reveals that somas located in the M2 channel (red) are also visible in the SST channel (green).

We found approximately 70 SST neurons in a

50-micron thick section from one hemisphere

of cortical V1 in mice stained for SST and M2

(slices from $\mathrm{n}=4$ mice, $\mu=69.8, \sigma=11.5$ ), $4 \%$

of which were also positive for M2 based on

colocalized antibody fluorescence. $73 \%$ of

M2-positive SST neurons were found in the

infragranular layers, with the remaining $27 \%$

in supragranular layers. All M2-positive

somas identified in V1 colocalized with SST

stain. We conclude from these experiments

that while $\mathrm{M} 2$ receptors are broadly expressed 
in V1, there is a subclass of SST neurons that might be of particular interest in future studies focused on spatiotemporal plasticity.

\section{Experience-dependent sequence potentiation occurs in monocular V1, with potentiation comparable to}

\section{binocular V1.}

In the immunofluorescence experiments above, we observed that the level of M2 expression differed between

binocular and monocular L4 of mouse

V1 (Fig. 3B). Reasoning that sequence

potentiation might scale with receptor

expression density, we hypothesized

that monocular V1 would show more

plasticity than binocular V1. To test

this, we implanted electrodes in left

monocular cortex (LMC) and right

binocular cortex (RBC) and modified

our stimulation setup to isolate the

LMC to determine whether visual

stimulation would drive potentiation

similar to that previously characterized

in binocular areas of V1. Covering the

left eye assured that all VEPs would

originate in the right eye, but since we

wanted to compare plasticity in LMC to

$\mathrm{RBC}$ it was also necessary to prevent

visual stimulation of ipsilateral

projections from the right eye (Hooks \&

Chen, 2020). To do this, we translated

the stimulus presentation screen right
A.
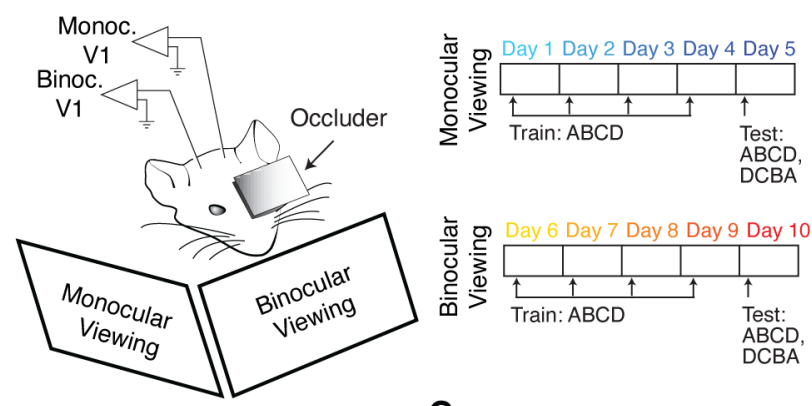

B.
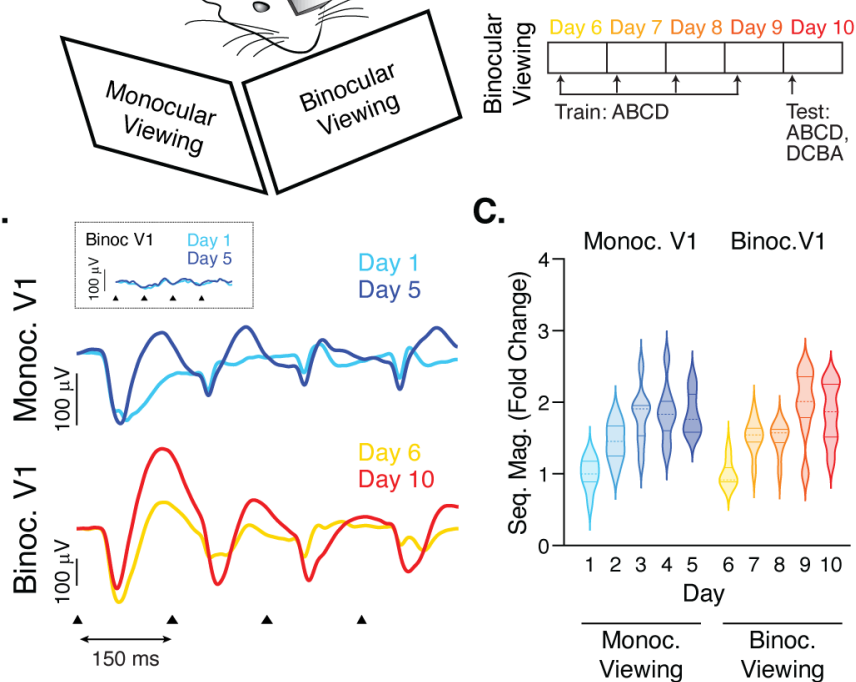

C.

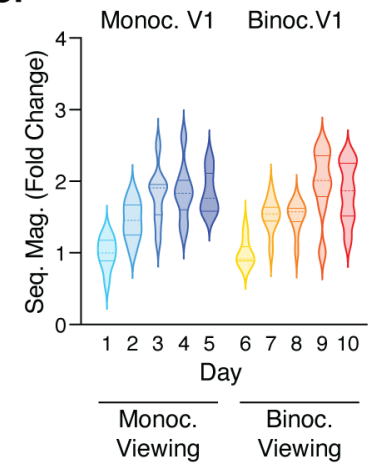

Figure 4 Monocular V1 is capable of spatiotemporal sequence learning and the extent of potentiation is similar to binocular V1. A. Animals $(\mathrm{n}=8)$ were implanted with electrodes in monocular V1 in the left hemisphere and binocular V1 in the right. For days 1-5, stimuli were presented only to the monocular cortex (contralateral projections from the right eye were isolated by placing a visual occluder over the left eye and positioning the screen at an angle of approximately $45^{\circ}$ off the right side of the snout). For days 6-10 both eyes were open, and the screen was positioned directly in front of the nose. B. Monocular stimulation (top) evoked smaller responses than did binocular stimulation (bottom, note scale bar differences) though in both cases the responses potentiated with training. The inset shows LFP recorded in the binocular cortex during monocular training and confirms that evoked activity was largely restricted to the left monocular cortex. C. Violin plots showing evoked responses in monocular (left) and binocular (right) cortex over training normalized by the average group magnitudes on days 1 (monoc.) and 6 (binoc.). Statistical analysis of fold changes using a Mixed model RM-ANOVA reveals that day is highly significant $\left(\mathrm{F}_{4,56}=43.058, \mathrm{p}<0.001\right)$ but that there is no effect of hemisphere $\left(\mathrm{F}_{1,14}=0.031, \mathrm{p}<0.864\right)$ or interaction between the two $\left(\mathrm{F}_{4,56}=2.279, \mathrm{p}=\right.$ 0.072 ). This result shows that monocular cortex is shaped by visual experience in a manner very similar to binocular cortex, but that the level of potentiation does not increase because of higher M2 receptor expression in monocular regions. 
and rotated it laterally until we saw no evoked potentials on the electrode implanted in the RBC (see Fig. 4A and the inset of 4B).

Animals were trained with this configuration, with visual responses isolated to the LMC, for four days and tested on day 5. The VEPs recorded in the monocular cortex were similar in shape but smaller in magnitude than those recorded previously in binocular V1, and their magnitude clearly increased with training (Fig. 4B top, though also see Fig. S3A-B). Starting on day 6, the visual occluder was removed and the screen was repositioned directly in front of the animal so that visual stimulation would activate RBC. Training continued as before on days 6-9 and the animals were tested on day 10. As expected, responses in the binocular cortex potentiated normally (Fig. 4B bottom and S3CD). We next normalized quantified sequence magnitudes from monocular recordings by the average response on day 1 and binocular recordings by the average response on day 6 (training day 1 in both cases) to directly compare fold changes between the two cortical regions (Fig. 4C). Our analysis of this data discovered no statistical difference in potentiation between monocular and binocular cortex. These findings show that visual experience is sufficient to drive long-term changes in evoked response dynamics in the monocular regions of V1 very similar to those seen in binocular areas (which has not been reported before), with both areas showing similar learning dynamics that saturate at approximately 2-fold of untreated baseline. They also suggest that whatever functional difference the increased expression of M2 receptors plays in the monocular cortex it does not cause experience dependent plasticity to occur faster or with a larger magnitude.

\section{Discussion}

Cholinergic signaling through mAChRs has been implicated in multiple forms of temporal processing in both humans and animal models, including spatiotemporal sequence learning, interval-timing (Abner et al., 2001; Balci et al., 2008; Zhang et al., 2019), temporal gap detection and ordinal tasks (Caine et al., 1981; Ison \& Bowen, 2000), and auditory discrimination (Seki et al., 2001) amongst others. Most of these works have designed their experiments around scopolamine, which is a non-selective $\mathrm{mAChR}$ antagonist. This work is the first to isolate the $\mathrm{M} 2$ receptor as being required specifically for spatiotemporal plasticity. It accords with results from a report that VEP amplitude increases resulting from electrical stimulation of the basal forebrain paired with phase-reversing sinusoidal gratings can be prevented by blocking nicotinic AChRs and M2 receptors in rats, though this work also found a role for M1 and depending on the spatial frequency of the visual inputs (Kang et al., 2015). 
M2 receptors can be involved with temporal associative memory tasks via their regulation of beta-site APPcleaving enzyme 1 (BACE1) expression, demonstrated by decreased freezing behavior in trace fear conditioning experiments with mice lacking this enzyme (Ohno et al., 2006; Roßner et al., 2006). M2 knockout mice show performance deficits in different spatial learning tasks such as the Barnes circular maze and T-maze delayed alternation task, including a lack of short-term potentiation and reduced long-term potentiation in the hippocampus after high frequency stimulation that was restored by a $\mathrm{GABA}_{\mathrm{A}}$ receptor antagonist (Seeger et al., 2004). This, along with a similar role for $\mathrm{GABA}_{\mathrm{A}} \mathrm{R}$ in Kang et al., 2015 suggests that the M2 receptor's functional role involves regulating GABAergic inhibition. Assuming this mechanism holds in V1, it would follow that sequence-evoked potentiation seen in the LFP results in part from dis-inhibition mediated increases in cortical sensitivity rather than increases in excitatory synapses.

Our identification of somatic M2 expression in SST+ neurons suggest that these cells in particular may play a role in this process. One possibility is that M2 receptor mediated plasticity modulates SST neurons to implement a "temporal surround suppression", akin to their role in spatial suppression (Niell and Scanziani, 2021), that biases responses towards learned spatiotemporal patterns. It should be noted, however, that excitatory neurons and other inhibitory neurons could also play an important role in this process. For example, M2AChR expression has been localized to PV+ cells in the rat entorhinal cortex using RNA amplification following single cell dissection (Chaudhuri et al., 2005) and there is every reason to expect that some of the M2 receptors we see in the neuropil are located on PV neurites. There are a myriad of other mechanisms that could be in play as well. Cholinergic activity enhances thalamocortical inputs, improves attention, etc. (Sarter and Parikh, 2005; Sarter et al., 2005; Groleau et al., 2015) and any of these processes could be modified by inhibitory activity. M2 receptors can hyperpolarize the neuronal membrane by opening GIRK (inward rectifying $\mathrm{K}^{+}$) channels (Brown, 2010) and they can also prevent the release of GABA from GABAergic terminals in rats (Salgado et al., 2007). Given the relation between mAChR mediated plasticity and GABA receptors found in other studies, it will be important to identify the exact role M2 plays in V1 to clarify its role in shaping temporally specific plasticity.

One interesting question is the overlap between scopolamine induced amnesia (Caine et al., 1981) and hippocampal involvement in memory engram formation. It is well established that the hippocampus plays a critical role in the formation of long-term memories, and it has also been shown that damage to the medial temporal lobe can prevent sequence potentiation in V1 (Finnie et al., 2021). Our findings suggest either that damage to the hippocampus 
prevents sequence learning in V1 by disrupting cholinergic projections, or that M2-mediated plasticity local to V1 allows the visual circuits to. Given the recently discovered modulation of V1 dynamics by hippocampal place representations (Saleem et al., 2018; Niell and Scanziani, 2021), it will be important to determine the extent to which local cortical circuits are shaped acutely by activity in hippocampus, indirectly by hippocampal-dependent activity of neuromodulators like ACh, and as a consequence of hippocampal-dependent consolidation during sleep (ReyesResina et al., 2021).

A variety of other studies have also reported M2 receptors in interneurons (Volpicelli and Levey, 2004; Groleau et al., 2015). M2 receptors specifically have been found in SST positive neurons in the rat amygdala, rat hippocampus and rat auditory cortex (Hajos et al., 1997; McDonald \& Mascagni, 2011; Salgado et al., 2007), and non-specific colocalization of mAChRs with SST has also been reported previously in mouse V1 (Chen et al., 2015). Interestingly, mAChRs are involved in mismatch negativity (MMN) in the auditory system (Pekkonen et al., 2001; Schöbi et al., 2021; Weber et al., 2021) and SST neurons have been implicated in visual perception in mice (Song et al., 2020) and in mismatch detection in the visual system (Hamm and Yuste, 2016; Attinger et al., 2017). MMN is an electrophysiological response generated in the brain when an expected stimulus is replaced by a deviant or oddball stimulus, and while the timeframe of this effect is quite different from the multi-day plasticity experiments used here there may be a relation in how cholinergic modulation and inhibitory circuits recognize novelty. Reduced MMN during the processing of sensory stimuli is a hallmark of schizophrenia (Baldeweg et al., 2006; Inami et al., 2008; Garrido et al., 2009; Inami and Kirino, 2019) and reduction of SST mRNA and mislocalization of SST neurons has also been observed in people with schizophrenia (Morris et al., 2008; Urban-Ciecko and Barth, 2016). SST neurons form synapses on both Vasoactive Intestinal Peptide (VIP) interneurons and pyramidal excitatory neurons, and a recent study focused on the role of visuomotor experience in shaping predictive coding in V1 found specific roles for both SST and VIP cells in signaling expectation violations (Attinger et al., 2017). Cumulatively, these studies highlight the role of muscarinic signaling in temporal predictive processes, may indicate a direct link between mAChR signaling in SST neurons and MMN suppression in schizophrenia, and possibly represent a mechanistic locus that could be probed in V1 circuits.

\section{Detailed Methods}


Animals. Male C57BL/6 mice (Charles River Laboratories) were group housed with littermates (four mice per cage) on a 12-h light/dark cycle and provided food and water ad libitum. Experimental and control groups were always selected randomly from littermates and yoked throughout the experiment. All experiments were performed during the light-cycle and animals were used for a single experiment only. All procedures were approved by the Institutional Animal Care and Use Committee of Boston University.

Electrode implantation. Mice were anesthetized with an intraperitoneal injection of $50 \mathrm{mg}$ per kg ketamine and 10 mg per kg xylazine and prepared for chronic recording. To facilitate head restraint, a steel headpost was affixed to the skull anterior to bregma using cyanoacrylate glue. Small $(<0.5 \mathrm{~mm})$ burr holes were drilled over binocular visual cortex (3 mm lateral from lambda) or monocular visual cortex $(\approx 2.2 \mathrm{~mm}$ lateral from lambda) and tungsten microelectrodes (for field recordings, FHC) were placed $450 \mu \mathrm{m}$ below the cortical surface. In all cases, a reference electrode (silver wire, A-M systems) was placed below dura over parietal cortex. All electrodes were rigidly secured to the skull using cyanoacrylate glue. Dental cement was used to enclose exposed skull and electrodes in a protective head cap. Buprenex (0.1 mg per kg) was injected subcutaneously for postoperative pain amelioration. Surgery was performed around postnatal day 45. Mice were monitored for signs of infection and allowed at least $24 \mathrm{~h}$ of recovery before habituation to the recording and restraint apparatus and were excluded from experiments only in the event of unsuccessful electrode implantation (lack of visual response, incorrect depth, poor grounding, etc.).

Stimulus presentation. Visual stimuli were generated using custom software written in Matlab (MathWorks) using the PsychToolbox extension (http://psychtoolbox.org/) to control stimulus drawing and timing. Sequences were constructed of four elements and an inter-sequence gray period. Each element consisted of a full-screen oriented highcontrast sinusoidal grating ( 0.5 cycles per deg). Sequence elements were separated by a minimum of 30 degrees and the order was restricted to prevent the appearance of rotation. Grating stimuli spanned the full range of monitor display values between black and white, with gamma correction to insure a linear gradient and constant total luminance in both gray-screen and patterned stimulus conditions. During experiments, animal handling involved placing each mouse (regardless of group membership, of which the investigator was aware) into the head-fixed presentation apparatus. Each sequence was presented 200 times per day in four groups of 50 presentations with each group separated by $30 \mathrm{~s}$. In the monocular versus binocular sequence learning experiment, monocular cortex was isolated by offsetting the screen to the right and adjusting it to an approximately $45^{\circ}$ angle relative to the mouse's midline while 
also covering the left eye with an opaque occluder for the first 5 days. Isolation was confirmed by showing brief flashing visual stimuli and verifying evoked responses were evident only in the in left monocular cortex (with the screen directly in front of the mouse, ipsilateral projections from the right eye drive responses in the right binocular cortex). For binocular training on days 6-10 the screen was placed directly in front of the mice and both eyes were open.

Data recording, analysis, and presentation. All data was amplified and digitized using the digital Recorder-64 system (Plexon). Local field potentials (LFPs) were recorded with 1-kHz sampling and a 200-Hz low-pass filter. LFP voltage traces in all figures show the average response of all mice in an experimental cohort. Data was extracted from the binary storage files and analyzed using custom software written in $\mathrm{C}++$ and Matlab, all of which is available for download at gavorniklab.bu.edu/supplemental-materials. To quantify plasticity effects sequence magnitude was defined as the average response magnitude (algorithmically scored peak-to-peak within the first $150 \mathrm{~ms}$ of visual stimulus onset) for each of the four elements in a sequence.

Statistics. All statistics were performed using the SPSS software package. Unless otherwise noted, comparisons between groups were made using a mixed model repeated measures ANOVA (RM-ANOVA), where session (e.g., recording day) was compared within subject groups assuming sphericity (confirmed with Mauchly's test) and treatment was a between subject variable. When main effects or interaction terms were significant, pairwise comparisons were performed using the Bonferroni correction for multiple comparisons. When making direct comparisons between groups, as when comparing ABCD to DCBA in supplemental materials, two-tailed pairedsample t-tests were used to calculate significance. The size of experimental cohorts was planned based on previously published experiments and our own experience which indicates that a minimum of 5 animals is needed in each cohort to reach statistical significance with adequate power (in most cases, more mice were used as a hedge against attrition), and we used power estimates in SPSS to verify that all statistically significant effects were $\geq 0.8$ for $\alpha=0.05$ (actual observed power was 1.0 for all statistically significant comparisons).

VU 0255035 injections. For the systemic VU 0255035 experiment, experimental mice were intraperitoneally injected with $10 \mathrm{mg}$ per kg of VU 0255035 (R\&D SYSTEMS) in DMSO + saline. Vehicle control animals were injected with DMSO + saline. Injections were performed 30 min before sequence presentation on experimental days $1-4$. This 
procedure was chosen to match previously published reports demonstrating that this dose is sufficient to effectively block M1 receptors in vivo (Sheffler et al, 2009; Crans et. al, 2020). To allow sufficient time for drug washout, recordings on the 5 th day occurred at least $24 \mathrm{~h}$ after the last injection on day 4.

AQ-RA 741 infusions. For the local AQ-RA 741 experiment, a 26-gauge guide cannula (Plastics One) was implanted lateral to the recording electrode in one hemisphere. The guide cannula was angled at $45^{\circ}$ relative to the recording electrode and positioned slightly below the cortical surface. Guides were affixed to the skull with cyanoacrylate and encased in the dental cement head cap and dummy cannulae were installed. After several days of recovery and habituation to the head-fixed restraints, the dummy cannulae were removed and infusion cannulae were lowered into the guides (the tip of the infusion cannulae were within $1 \mathrm{~mm}$ of the recording electrode tip; see schematic in Fig. 2A). A Nanoject II (Warner Instruments), under the remote control of custom software, was used to infuse $1 \mu$ l of vehicle (DMSO + saline) or $1 \mu \mathrm{l}$ of AQ-RA 741 (R\&D SYSTEMS) $\left(0.2 \mathrm{mg} \mathrm{ml}^{-1}, 1 \mathrm{mg}\right.$ of the drug in DMSO + saline) in 2.3-nl pulses evenly spread over 10 mins $\left(6 \mu \mathrm{h}^{-1}\right.$ infusion rate) before stimulus presentation on experimental days 1 and 2 under isoflurane anesthesia. Animals were recorded 25 min after infusion completion to ensure full recovery from the anesthesia. Mice received either drug or vehicle infusions, and all mice that evidenced clear visual responsiveness in both hemispheres (that is, no trauma caused by infusion) were included in the study. Local treatment was performed for only $2 \mathrm{~d}$ to minimize inherent cortical trauma associated with repeated insertions and removals of the infusion and dummy cannulae; training continued for 2 additional days after drug washout to verify that V1 was rendered aplastic because of drug treatment and not an unintentional cortical lesion.

Immunofluorescence. Animals were intraperitoneally injected with pentobarbital (100 mg/kg, Fatal Plus, Covetrus, 035946) and then perfused with $4 \%$ paraformaldehyde (PFA) in PBS. Fixed tissue was cryoprotected in 30g\% sucrose and sectioned via cryostat to obtain coronal sections (50 $\mu \mathrm{m}$ slices). Sections were permeabilized and blocked using a solution of $0.1 \%$ Triton-X, $10 \%$ NGS, $1 \%$ BSA in PBS. Sections were incubated with primary antibodies against M2 muscarinic receptor (rat anti-M2, Millipore, MAB367) and PV (rabbit anti-PV, Abcam, ab11427) or SST (rabbit antiSST, Fisher Scientific, NBP1-87022) neuronal proteins at a dilution of 1:500 each in 10\% NGS in PBS overnight at $4^{\circ} \mathrm{C}$. Slices were next washed three times with $0.1 \%$ Triton-X in PBS for 5-10 minutes per wash, and then then incubated with secondary antibodies at 1:500 dilution in either 10\% NGS in PBS (goat anti-rat, Alexa Fluor 647, Thermo Fisher, A21247) or 1\% BSA in PBS (donkey anti-rabbit, Alexa Fluor 488, Thermo Fisher, A21206) at room 
temperature for 2 hours. For nuclear dsDNA staining, slices were incubated in a 1:10,000 dilution of Hoechst (Thermo Fisher, H1399) in 1\% BSA in PBS for 2 hours at room temperature. Following secondary antibody incubation, slices were rinsed three times in PBS for 5-10 minutes per rinse. Slices were then mounted to slides with Fluoro-gel mounting media. Fluorescence images were captured using a Nikon Eclipse Brightfield Microscope in the following channels: DAPI (Hoechst/blue), FITC (Alexa 488/green), CY5 (Alexa 647/red).

To quantify the number of PV+ and SST+ neurons, we used histological images from $50 \mu \mathrm{m}$ slices of a singlehemisphere of V1 (including monocular and binocular regions) and analyzed them in ImageJ using the following steps: 1. grayscale conversion 2. background subtraction 3. threshold adjustment to remove noise 4. conversion to black and white format 5. counting neurons. For PV/SST and M2 co-localization, we first counted the number of interneurons and M2 positive cells separately, then counted the number of cells which were positive for both the interneuron (green channel) and M2 (red channel) in an overlap image by looking for yellow cells (red-green overlap). Since the number of positive cells was low, it was easy to confirm each overlap manually in both the red and green channels.

To quantify M2 expression levels, histological images of M2 staining in mouse V1 (including monocular and binocular regions) were imported into MATLAB and converted to grayscale. The average pixel intensity of V1 was computed. Each image was then cropped into monocular and binocular V1 and processed independently. The row-wise mean of each monocular and binocular image was calculated, creating an array of values corresponding to the average pixel intensity by cortical depth. These values were normalized by the average pixel intensity of the corresponding V1 image (based on both monocular and binocular regions). The normalized pixel intensity values were averaged among different animals and plotted against cortical depth (values extracted from metadata in ImageJ) along with 95\% confidence intervals of the group mean. To compare expression levels in supragranular, granular and infragranular layers, mean intensity levels were calculated for $0-350,350-450$, and $450-700 \mu \mathrm{m}$ ranges using the same data.

\section{Conflict of Interest}

Nothing to declare 


\section{Author Contributions}

Conception and experimental design: S.S. and J.P.G. Surgeries, experiments and data collection: S.S. and C.M.J. Data analysis: S.S., C.R-M., C.M.J. and J.P.G. Writing and figures: S.S. and J.P.G.

\section{References}

Abner RT, Edwards T, Douglas A (2001) Pharmacology of Temporal Cognition in Two Mouse Strains. International Journal of Comparative Psychology 14:189-200.

Attinger A, Wang B, Keller GB (2017) Visuomotor Coupling Shapes the Functional Development of Mouse Visual Cortex. Cell 169:1291-1302.

Balci F, Ludvig EA, Gibson JM, Allen BD, Frank KM, Kapustinski BJ, Fedolak TE, Brunner D (2008) Pharmacological manipulations of interval timing using the peak procedure in male $\mathrm{C} 3 \mathrm{H}$ mice. Psychopharmacology 201:67-80.

Baldeweg T, Wong D, Stephan KE (2006) Nicotinic modulation of human auditory sensory memory: Evidence from mismatch negativity potentials. International Journal of Psychophysiology 59:49-58.

Brown DA (2010) Muscarinic acetylcholine receptors (mAChRs) in the nervous system: Some functions and mechanisms. Journal of Molecular Neuroscience 41:340-346.

Caine ED, Weingartner H, Ludlow CL, Cudahy EA, Wehry S (1981) Qualitative Analysis of Scopolamine-Induced Amnesia. Psychopharmacology 74:74-80.

Chaudhuri J, Hiltunen M, Nykänen M, Ylä-Herttuala S, Soininen H, Miettinen R (2005) Localization of M2 muscarinic receptor protein in parvalbumin and calretinin containing cells of the adult rat entorhinal cortex using two complementary methods. Neuroscience 131:557-566.

Chen N, Sugihara H, Sur M (2015) An acetylcholine-activated microcircuit drives temporal dynamics of cortical activity. Nature Neuroscience 18:892-902.

Chubykin AA, Roach EB, Bear MF, Shuler MGH (2013) A Cholinergic Mechanism for Reward Timing within Primary Visual Cortex. Neuron 77:723-735.

Collins AL, Aitken TJ, Greenfield VY, Ostlund SB, Wassum KM (2016) Nucleus accumbens acetylcholine receptors modulate dopamine and motivation. Neuropsychopharmacology 41:2830-2838. 
Coppola JJ, Disney AA (2018) Is there a canonical cortical circuit for the cholinergic system? Anatomical differences across common model systems. Frontiers in Neural Circuits 12:1-13.

Crans RAJ, Wouters E, Valle-Leon M, Taura J, Massari CM, Fernandez-Duenas V, Stove CP, Ciruela F (2020) Striatal Dopamine D2-Muscarinic Acetylcholine M1 Receptor-Receptor Interaction in a Model of Movement Disorders. Frontiers in Pharmacology 11:1-13.

David Weaver C, Sheffler DJ, Lewis LM, Bridges TM, Williams R, Nalywajko NT, Kennedy JP, Mulder MM, Jadhav S, Aldrich LA, Jones CK, Marlo J, Niswender CM, Mock MM, Conn PJ, Lindsley CW (2009) Discovery and development of a potent and highly selective small molecule muscarinic acetylcholine receptor subtype I (mAChR 1 or M1 ) antagonist in vitro and in vivo probe. Current Topics in Medicinal Chemistry 9:1217-1226.

Doods H, Entzeroth M, Mayer N (1991) Cardioselectivity of AQ-RA 741, a novel tricyclic antimuscarinic drug. European Journal of Pharmacology 192:147-152.

Doods HN, Quirion R, Mihm G, Engel W, Rudolf K, Entzeroth M, Schiavi GB, Ladinsky H, Bechtel WD, Ensinger HA, Mendla KD, Eberlein W (1993) Therapeutic Potential of Cns-Active M 2 Antagonists: Novel Structures and Pharmacology. Life Sciences 52:497-503.

Dorje F, Wess J, Lambrecht G, Tacke R, Mutschler E, Brann3 MR (1991) Antagonist Binding Profiles of Five Cloned Human Muscarinic Receptor Subtypes1. The journal of pharmacology and experimental therapeutics 256:727-733.

Elhusseiny A, Hamel E (2000) Muscarinic-but Not Nicotinic-Acetylcholine Receptors Mediate a Nitric OxideDependent Dilation in Brain Cortical Arterioles: A Possible Role for the M5 Receptor Subtype. Journal of cerebral flood flow and metabolism 20:298-305.

Finnie PSB, Komorowski RW, Bear MF (2021) The spatiotemporal organization of experience dictates hippocampal involvement in primary visual cortical plasticity. Current Biology 31:3996-4008.

Floody OR (2014) Role of acetylcholine in control of sexual behavior of male and female mammals. Pharmacology Biochemistry and Behavior 120:50-56.

Garrido MI, Kilner JM, Stephan KE, Friston KJ (2009) The mismatch negativity: A review of underlying mechanisms. Clinical Neurophysiology 120:453-463. 
Gavornik JP, Bear MF (2014) Learned spatiotemporal sequence recognition and prediction in primary visual cortex. Nature Neuroscience 17:732-737.

Goard M, Dan Y (2009) Basal forebrain activation enhances cortical coding of natural scenes. Nature Neuroscience 12:1444-1449.

Groleau M, Kang J il, Huppé-Gourgues F, Vaucher E (2015) Distribution and effects of the muscarinic receptor subtypes in the primary visual cortex. Frontiers in Synaptic Neuroscience 7:1-9.

Gu Q (2003) Contribution of acetylcholine to visual cortex plasticity. Neurobiology of Learning and Memory 80:291-301.

Hamm JP, Yuste R (2016) Somatostatin Interneurons Control a Key Component of Mismatch Negativity in Mouse Visual Cortex. Cell Reports 16:597-604.

Hasselmo ME (2006) The role of acetylcholine in learning and memory. Current Opinion in Neurobiology 16:710715.

Hasselmo ME, McGaughy J (2004) High acetylcholine levels set circuit dynamics for attention and encoding, and low acetylcholine levels set dynamics for consolidation. Progress in Brain Research 145:207-231.

Herman AM, Ortiz-Guzman J, Kochukov M, Herman I, Quast KB, Patel JM, Tepe B, Carlson JC, Ung K, Selever J, Tong Q, Arenkiel BR (2016) A cholinergic basal forebrain feeding circuit modulates appetite suppression. Nature 538:253-256.

Herrero JL, Roberts MJ, Delicato LS, Gieselmann MA, Dayan P, Thiele A (2008) Acetylcholine contributes through muscarinic receptors to attentional modulation in V1. Nature 454:1110-1114.

Bubser M, Byun N, Wood MR, Jones CK (2012) Muscarinic receptor pharmacology and circuitry for the modulation of cognition. Handbook of Experimental Pharmacology 208:121-66.

Hooks BM, Chen C (2020) Circuitry Underlying Experience-Dependent Plasticity in the Mouse Visual System. Neuron 106:21-36.

Shuler MG, Bear MF (2006) Reward timing in the primary visual cortex. Science 311:1606-1609.

Inami R, Kirino E (2019) Nicotine effect on mismatch negativity in smoking and nonsmoking patients with schizophrenia. Schizophrenia Research 208:496-497.

Inami R, Kirino E, Inoue R, Suzuki T, Arai H (2008) Nicotine effects on mismatch negativity in nonsmoking schizophrenic patients. Neuropsychobiology 56:64-72. 
Ison JR, Bowen GP (2000) Scopolamine reduces sensitivity to auditory gaps in the rat, suggesting a cholinergic contribution to temporal acuity. Hearing Research 145:169-176.

Ji W, Gămănuţ R, Bista P, D’Souza RD, Wang Q, Burkhalter A (2015) Modularity in the Organization of Mouse Primary Visual Cortex. Neuron 87:632-643.

Jos NH, Papp EC, Acsa LD, Levey AI, Freund TF (1997) Distinct Interneuron Types Express M2 Muscarinic Receptor Immunoreactivity on Their Dendrites or Axon Terminals in The Hippocampus. Neuroscience 82:355376.

Kang J il, Huppé-Gourgues F, Vaucher E (2015) Pharmacological mechanisms of cortical enhancement induced by the repetitive pairing of visual/cholinergic stimulation. PLoS ONE 10:1-18.

Lee SH, Dan Y (2012) Neuromodulation of Brain States. Neuron 76:209-222.

Levey AI, Edmund SM, Heilman CJ, Desmonds TI, Frey KA (1994) Localization of Muscarinic M3 Receptor Protein and M3 Receptor Binding In Rat Brain. Neuroscience 63:207-221.

Levey AI, Kitt CA, Simonds WF, Price DL, Brann MR (1991) Identification and localization of muscarinic acetylcholine receptor proteins in brain with subtype-specific antibodies. Journal of Neuroscience 11:32183226.

Lewis AS, Pittenger ST, Mineur YS, Stout D, Smith PH, Picciotto MR (2018) Bidirectional Regulation of Aggression in Mice by Hippocampal Alpha-7 Nicotinic Acetylcholine Receptors. Neuropsychopharmacology 43:1267-1275.

Liu CH, Coleman JE, Davoudi H, Zhang K, Hussaina Shuler MG (2015) Selective Activation of a Putative Reinforcement Signal Conditions Cued Interval Timing in Primary Visual Cortex. Current Biology 25:15511561.

Lochner M, Thompson AJ (2016) The muscarinic antagonists scopolamine and atropine are competitive antagonists at 5-HT3 receptors. Neuropharmacology 108:220-228.

Mancillas JR, Sigginst GR, Bloom FE (1986) Somatostatin selectively enhances acetylcholine-induced excitations in rat hippocampus and cortex. PNAS 83:7518-7521.

McDonald AJ, Mascagni F (2011) Neuronal localization of M2 muscarinic receptor immunoreactivity in the rat amygdala. Neuroscience 196:49-65. 
Miyakawa T, Yamada M, Duttaroy A, Rgen Wess J (2001) Hyperactivity and Intact Hippocampus-Dependent Learning in Mice Lacking the M 1 Muscarinic Acetylcholine Receptor. The Journal of Neuroscience 21:52395250.

Morris HM, Hashimoto T, Lewis DA (2008) Alterations in somatostatin mRNA expression in the dorsolateral prefrontal cortex of subjects with schizophrenia or schizoaffective disorder. Cerebral Cortex 18:1575-1587.

Mrzljak L, Leveyt AI, Goldman_Rakic PS (1993) Association of $\mathrm{ml}$ and $\mathrm{m} 2$ muscarinic receptor proteins with asymmetric synapses in the primate cerebral cortex: Morphological evidence for cholinergic modulation of excitatory neurotransmission. PNAS 90:5194-5198.

Muñoz W, Rudy B (2014) Spatiotemporal specificity in cholinergic control of neocortical function. Current Opinion in Neurobiology 26:149-160.

Namboodiri VMK, Huertas MA, Monk KJ, Shouval HZ, Shuler MGH (2015) Visually cued action timing in the primary visual cortex. Neuron 86:319-330.

Niell CM, Scanziani M (2021) How Cortical Circuits Implement Cortical Computations: Mouse Visual Cortex as a Model. Annual Review of Neuroscience 44:517-546.

Ohno M, Chang L, Tseng W, Oakley H, Citron M, Klein WL, Vassar R, Disterhoft JF (2006) Temporal memory deficits in Alzheimer's mouse models: Rescue by genetic deletion of BACE1. European Journal of Neuroscience 23:251-260.

Parikh V, Kozak R, Martinez V, Sarter M (2007) Prefrontal acetylcholine release controls cue detection on multiple time scales. Neuron 56:141-154.

Pekkonen E, Hirvonen J, Jääskeläinen IP, Kaakkola S, Huttunen J (2001) Auditory sensory memory and the cholinergic system: Implications for Alzheimer's disease. NeuroImage 14:376-382.

Pfeffer CK, Xue M, He M, Huang ZJ, Scanziani M (2013) Inhibition of inhibition in visual cortex: The logic of connections between molecularly distinct interneurons. Nature Neuroscience 16:1068-1076.

Pi HJ, Hangya B, Kvitsiani D, Sanders JI, Huang ZJ, Kepecs A (2013) Cortical interneurons that specialize in disinhibitory control. Nature 503:521-524.

Pinto L, Goard MJ, Estandian D, Xu M, Kwan AC, Lee SH, Harrison TC, Feng G, Dan Y (2013) Fast modulation of visual perception by basal forebrain cholinergic neurons. Nature Neuroscience 16:1857-1863. 
Reyes-Resina I, Samer S, Kreutz M, Oelschlegel A (2021) Molecular Mechanisms of Memory Consolidationt that Operate During Sleep. Frontiers in Molecular Neuroscience 14:1-21.

Roßner S, Sastre M, Bourne K, Lichtenthaler SF (2006) Transcriptional and translational regulation of BACE1 expression-Implications for Alzheimer's disease. Progress in Neurobiology 79:95-111.

Ruzzoli M, Pirulli C, Mazza V, Miniussi C, Brignani D (2016) The mismatch negativity as an index of cognitive decline for the early detection of Alzheimer's disease. Scientific Reports 6:1-11.

Saleem AB, Diamanti EM, Fournier J, Harris KD, Carandini M (2018) Coherent encoding of subjective spatial position in visual cortex and hippocampus. Nature 562:124-127.

Salgado H, Bellay T, Nichols JA, Bose M, Martinolich L, Perrotti L, Atzori M (2007) Muscarinic M2 and M1 receptors reduce GABA release by $\mathrm{Ca} 2+$ channel modulation through activation of $\mathrm{PI} 3 \mathrm{~K} / \mathrm{Ca} 2$ +-independent and PLC/Ca2+-dependent PKC. Journal of Neurophysiology 98:952-965.

Sarter M, Hasselmo ME, Bruno JP, Givens B (2005) Unraveling the attentional functions of cortical cholinergic inputs: Interactions between signal-driven and cognitive modulation of signal detection. Brain Research Reviews 48:98-111.

Sarter M, Parikh V (2005) Choline transporters, cholinergic transmission and cognition. Nature Reviews Neuroscience 6:48-56.

Saunders DS, Saunders JS, Pittendngh DS, Minis CS, Amer DH (1969) Effect of intrahypothalamic infusion of acetylcholine on behavioral and physiological thermoregulation in the rat. Nature 221:561-562.

Schöbi D, Homberg F, Frässle S, Endepols H, Moran RJ, Friston KJ, Tittgemeyer M, Heinzle J, Stephan KE (2021) Model-based prediction of muscarinic receptor function from auditory mismatch negativity responses. NeuroImage 237:1-13.

Seeger T, Fedorova I, Zheng F, Miyakawa T, Koustova E, Gomeza J, Basile AS, Alzheimer C, Wess J (2004) M2 muscarinic acetylcholine receptor knock-out mice show deficits in behavioral flexibility, working memory, and hippocampal plasticity. Journal of Neuroscience 24:10117-10127.

Sheffler DJ, Williams R, Bridges TM, Xiang Z, Kane AS, Byun NE, Satyawan Jadhav S, Mock MM, Zheng F, Lewis LM, Jones CK, Niswender CM, Weaver CD, Lindsley CW, Conn PJ (2009) A novel selective muscarinic acetylcholine receptor subtype 1 antagonist reduces seizures without impairing hippocampusdependent learning. Molecular Pharmacology 76:356-368. 
Sidorov MS, Kim H, Rougie M, Williams B, Siegel JJ, Gavornik JP, Philpot BD (2020) Visual Sequences Drive Experience-Dependent Plasticity in Mouse Anterior Cingulate Cortex. Cell Reports 32:1-12.

Seki K, Kudoh M, Shibuki K (2001) Sequence dependence of post-tetanic potentiation after sequential heterosynaptic stimulation in the rat auditory cortex. Journal of Physiology 533.2:503-518.

Song Y-H, Hwang Y-S, Kim K, Lee H-R, Kim J-H, Maclachlan C, Dubois A, Jung MW, Petersen CCH, Knott G, Lee S-H, Lee S-H (2020) Somatostatin enhances visual processing and perception by suppressing excitatory inputs to parvalbumin-positive interneurons in V1. Science Advances 6:1-11.

Steriade M (2004) Acetylcholine systems and rhythmic activities during the waking-sleep cycle. Progress in Brain Research 145:179-196.

Thiele A (2013) Muscarinic signaling in the brain. Annual Review of Neuroscience 36:271-294.

Urban-Ciecko J, Barth AL (2016) Somatostatin-expressing neurons in cortical networks. Nature Reviews Neuroscience 17:401-409.

Vaucher E, Reymond I, Najaffe R, Kar S, Quirion R, Miller MM, Franklin KBJ (2002) Estrogen effects on object memory and cholinergic receptors in young and old female mice. Neurobiology of Aging 23:87-95.

Volpicelli LA, Levey AI (2004) Muscarinic acetylcholine receptor subtypes in cerebral cortex and hippocampus. Progress in Brain Research 145:59-66.

Bear MF, Singer W (1986) Modulation of visual cortical plasticity by acetylcholine and noradrenaline. Nature $320: 172-176$.

Weaver CD, Sheffler DJ, Lewis LM, Bridges TM, Williams R, Nalywajko NT, Kennedy JP, Mulder MM, Jadhav S, Aldrich LA, Jones CK, Marlo J, Niswender CM, Mock MM, Zheng F, P. Conn J, Lindsley CW (2009) Discovery and development of a potent and highly selective small molecule muscarinic acetylcholine receptor subtype I (mAChR 1 or M1) antagonist in vitro and in vivo probe. 9:1217-1226

Weber LA, Tomiello S, Schöbi D, Wellstein K v, Müller D, Iglesias S, Stephan KE (2021) Auditory mismatch responses are differentially sensitive to changes in muscarinic acetylcholine versus dopamine receptor function. bioRxiv preprint doi: https://doi.org/10.1101/2021.03.18.435979

Winkler I (2007) Interpreting the mismatch negativity. Journal of Psychophysiology 21:147-163.

Zhang Q, Jung D, Larson T, Kim Y, Narayanan NS (2019) Scopolamine and Medial Frontal Stimulus-Processing during Interval Timing. Neuroscience 414:219-227. 
Zhang W, Basile AS, Gomeza J, Volpicelli LA, Levey AI, Rgen Wess J (2002) Characterization of Central

Inhibitory Muscarinic Autoreceptors by the use of Muscarinic Acetylcholine Receptor Knock-Out Mice. The Journal of Neuroscience. 22:1709-1717. 